\title{
Towards Low-Latency and Ultra-Reliable Vehicle-to-Vehicle Communication
}

\author{
Muhammad Ikram Ashraf*, Chen-Feng Liu*, Mehdi Bennis*†, and Walid Saad ${ }^{\ddagger}$ \\ ${ }^{*}$ Centre for Wireless Communications, University of Oulu, Finland \\ ${ }^{\dagger}$ Department of Computer Engineering, Kyung Hee University, South Korea \\ ${ }^{\ddagger}$ Wireless@ VT, Bradley Department of Electrical and Computer Engineering, Virginia Tech, Blacksburg, VA, USA \\ E-mail: \{ikram.ashraf, chen-feng.liu, mehdi.bennis $\} @$ oulu.fi, walids@vt.edu
}

\begin{abstract}
Recently vehicle-to-vehicle (V2V) communication emerged as a key enabling technology to ensure traffic safety and other mission-critical applications. In this paper, a novel proximity and quality-of-service $(\mathrm{Q} o S)$-aware resource allocation for $\mathrm{V} 2 \mathrm{~V}$ communication is proposed. The proposed approach exploits the spatial-temporal aspects of vehicles in terms of their physical proximity and traffic demands, to minimize the total transmission power while considering queuing latency and reliability. Due to the overhead caused by frequent information exchange between vehicles and the roadside unit (RSU), the centralized problem is decoupled into two interrelated subproblems. First, a novel RSU-assisted virtual clustering mechanism is proposed to group vehicles in zones based on their physical proximity. Given the vehicles' traffic demands and their QoS requirements, resource blocks are assigned to each zone. Second, leveraging techniques from Lyapunov stochastic optimization, a power minimization solution is proposed for each $\mathrm{V} 2 \mathrm{~V}$ pair within each zone. Simulation results for a Manhattan model have shown that the proposed scheme outperforms the baseline in terms of average queuing latency reduction up to $97 \%$ and significant improvement in reliability.
\end{abstract}

Index Terms-5G, ultra-reliable low latency communications (URLLC), vehicle-to-vehicle (V2V) communications.

\section{INTRODUCTION}

Vehicle-to-vehicle (V2V) communication is envisioned as one of the most promising enablers for intelligent transportation systems (ITSs) [1]. Although some applications in V2V communication, e.g., lane change alerts and automatic braking, have already been deployed, safety concerns in autonomous transportation and other mission-critical applications still pose significant challenges for vehicular networks. V2V safety services aim at reducing the risk of traffic accidents. In this regard, ETSI has standardized two safety messages: cooperative awareness message (CAM) and decentralized environmental notification message (DENM) [2]. Transmitting these messages with ultra reliability and low latency is a crucial requirement. Although IEEE 802.11p supports V2V communication, it suffers from unbounded latency and varying quality-ofservice (QoS) guarantees [3]. According to 5G requirements, a queuing latency of $0.125 \mathrm{~ms}$ is required in order to achieve $1 \mathrm{~ms}$ end-to-end latency [4].

Radio resource management (RRM) plays a key role in the performance of wireless vehicular systems, and it faces many new challenges in the context of stringent QoS-based V2V communications [5]. To address the RRM challenges, a number of recent works have emerged focusing on latency and reliability in $\mathrm{V} 2 \mathrm{~V}$ communication [6]-[8]. The authors in [6] propose a centralized heuristic QoS-based RRM for $\mathrm{V} 2 \mathrm{~V}$ communication focusing on a sum-rate maximization problem without accounting for the queue state information (QSI). A location-dependent resource allocation by enabling hierarchical clustering is proposed in [7] for V2V communication. Furthermore, full buffer traffic model is considered, and a subset of orthogonal resource blocks (RBs) are reserved for $\mathrm{V} 2 \mathrm{~V}$ communication within the same cell [7]. An RB sharing algorithm which limits the accumulated interference to ensure reliability of $\mathrm{V} 2 \mathrm{~V}$ communication is proposed in [8]. Most of the these works on $\mathrm{V} 2 \mathrm{~V}$ resource allocation focus on full buffer traffic and do not account for queuing latency, instantaneous traffic demand, and bounded queue size in order to reduce interference. Moreover, this prior art fails to address issues pertaining to the joint optimization of queuing latency and reliability for $\mathrm{V} 2 \mathrm{~V}$-based resource allocation [6]-[8].

The main contribution of this paper is a clean-slate design of resource allocation while considering queuing latency and reliability requirements for $\mathrm{V} 2 \mathrm{~V}$ communication. Unlike previous works such as [6] and [8], our proposed work jointly considers resource allocation and power control for interference mitigation while blending geographical information and queue dynamics. In order to capture QSI, each vehicle user equipment (VUE) transmitter uses a queue buffer to store data intended for its VUE receiver. The network problem is formulated as a network transmit power minimization problem subject to probabilistic realibility constraints on vehicle's data queue length. We further utilize Markov's inequality to transform the probabilistic constraint into a latency requirement in terms of average queue length. To solve the formulated problem, we propose a roadside unit (RSU)-assisted approach which decouples the problem into two sub-problems addressed at the RSU and VUE levels, separately. In the proposed scheme, the RSU groups pairs of vehicles into virtual zones within which each $\mathrm{V} 2 \mathrm{~V}$ pair can optimize its transmit power over the set of allocated RBs while satisfying latency and reliability requirements. Due to the localized nature of traffic safety applications, a new virtual zone formation approach is proposed based on the network topology, traffic demand, and latency requirements. By leveraging Lyapunov optimization techniques, a power minimization is addressed at the VUE while ensuring queuing latency requirements and reliability 


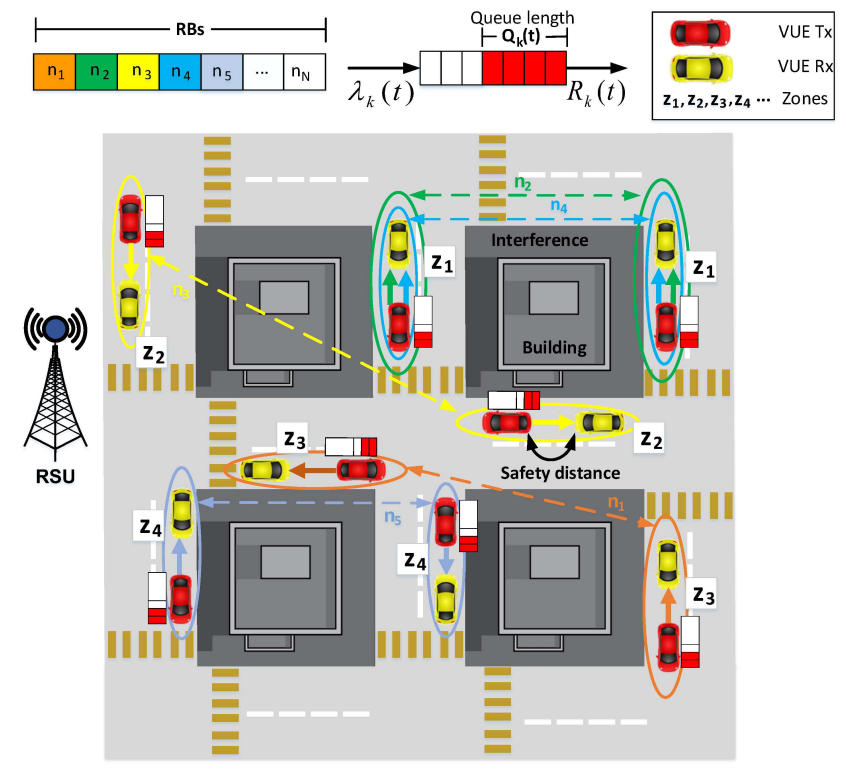

Figure 1. Considered Manhattan mobility model.

constraints. Simulation results validate the effectiveness and performance of the proposed approach as compared to a baseline in terms of queuing latency and high reliability.

\section{System Model and Problem Formulation}

\section{A. System Model}

Consider a Manhattan mobility model with V2V communication composed of $K$ VUE transmitter-receiver pairs under the coverage of a single RSU, as illustrated in Fig. 1 Every VUE pair configuration is fixed during the entire communication period. The safety distance between the transmitter and receiver of a single VUE pair is bounded in a region which is small compared with the considered network area. Let $\mathcal{K}$ be the set of all $K$ of VUE pairs who share a set $\mathcal{N}$ of $N$ orthogonal RBs with equal bandwidth $\omega$. Each VUE pair uses multiples RBs whereas one RB can be reused by multiple VUE pairs which causes interference. Communication is slotted and indexed by $t . h_{k k^{\prime}}^{n}(t)$ denotes the signal propagation channel from the transmitter of pair $k$ to the receiver of pair $k^{\prime}$ over RB $n$ in slot $t$. Full information of $h_{k k}^{n}(t), \forall t, n \in \mathcal{N}$, is assumed to be known at VUE pair $k$.

We define $\eta_{k}^{n}(t) \in\{0,1\}$ as the RB usage in time slot $t$ where $\eta_{k}^{n}(t)=1$ indicates that VUE pair $k$ uses RB $n$. Otherwise, $\eta_{k}^{n}(t)=0$. To send information to the receiver, the transmitter of pair $k$ allocates power $P_{k}^{n}(t)$ over RB $n$ in slot $t$ with $\sum_{n \in \mathcal{N}} \eta_{k}^{n}(t) P_{k}^{n}(t) \leq P_{k}^{\max }$ where $P_{k}^{\max }$ is the total power budget. The data rate of VUE pair $k$ in time slot $t$ is:

$$
R_{k}(t)=\sum_{n \in \mathcal{N}} \omega \log _{2}\left(1+\frac{\eta_{k}^{n}(t) P_{k}^{n}(t)\left|h_{k k}^{n}(t)\right|^{2}}{\sigma^{2}+\sum_{k^{\prime} \in \mathcal{K} \backslash k} \eta_{k^{\prime}}^{n}(t) P_{k^{\prime}}(t)\left|h_{k^{\prime} k}^{n}(t)\right|^{2}}\right),
$$

where $\sigma^{2}$ is the noise variance. Moreover, each VUE transmitter has a queue buffer to store data for its VUE receiver. Denoting VUE pair $k$ 's queue length at the beginning of slot $t$ as $Q_{k}(t)$, the evolution of $Q_{k}(t)$ is given by:

$$
Q_{k}(t+1)=\max \left\{Q_{k}(t)+\lambda_{k}(t)-\tau R_{k}(t), 0\right\} .
$$

Here, $\tau$ is the time slot duration while $\lambda_{k}(t)$, with $\bar{\lambda}_{k}=$ $\mathbb{E}\left[\lambda_{k}(t)\right]$, is the traffic arrival at the transmitter of pair $k$ in the beginning of slot $t$. Our goal is to consider resource allocation and power optimization while satisfying the latency and reliability requirements, in terms of queue length, needed to deliver the safety messages.

\section{B. Problem Formulation}

Our objective is to find an efficient joint resource and power allocation scheme while meeting the queuing latency and reliability requirements [4]. According to Little's law, the average queuing latency is proportional to the average queue length [9]. Additionally, when the finite-size queue buffer is overloaded, data arrival results in packet drops which incurs unreliable communication. Therefore, the queue length is a key parameter for modeling the queuing latency and reliability for V2V communication. To this end, we impose a probabilistic constraint on the queue length for each VUE pair $k \in \mathcal{K}$, i.e., $\operatorname{Pr}\left(Q_{k}(t) \geq L_{k}\right) \leq \epsilon_{k}$, with an allowable queue length $L_{k}$ and a tolerance value $\epsilon_{k} \ll 1$ considered as reliability. In our model, the power consumption for transmission is coupled with the resource usage. Hence, the RSU's objective is to find an optimal network-wide RB usage and power allocation strategy in order to minimize the time-averaged network power while satisfying the probabilistic constraint on queue length. Formally, denoting the network RB usage and power allocation vectors as $\boldsymbol{\eta}(t)=\left(\eta_{k}^{n}(t), k \in \mathcal{K}, n \in \mathcal{N}\right)$ and $\boldsymbol{P}(t)=\left(P_{k}^{n}(t), k \in \mathcal{K}, n \in \mathcal{N}\right), \forall t$, respectively, the network-wide optimization problem is written as follows:

$$
\begin{array}{cl}
\underset{\boldsymbol{\eta}(t), \boldsymbol{P}(t)}{\operatorname{minimize}} & \sum_{k \in \mathcal{K}} \sum_{n \in \mathcal{N}} \bar{P}_{k}^{n} \\
\text { subject to } & \lim _{t \rightarrow \infty} \operatorname{Pr}\left(Q_{k}(t) \geq L_{k}\right) \leq \epsilon_{k}, \forall k \in \mathcal{K}, \\
& \sum_{n \in \mathcal{N}} \eta_{k}^{n}(t) P_{k}^{n}(t) \leq P_{k}^{\max }, \forall t, k \in \mathcal{K}, \\
& P_{k}^{n}(t) \geq 0, \forall t, k \in \mathcal{K}, n \in \mathcal{N}, \\
& \eta_{k}^{n}(t) \in\{0,1\}, \forall t, k \in \mathcal{K}, n \in \mathcal{N},
\end{array}
$$

where $\bar{P}_{k}^{n}=\lim _{T \rightarrow \infty} \frac{1}{T} \sum_{t=1}^{T} P_{k}^{n}(t)$ is the time-averaged power consumption of VUE pair $k$ over RB $n$. Since it is challenging to analytically analyze (2b), we resort to Markov's inequality to linearize the probabilistic constraint.

Definition 1 (Markov's inequality). If $X$ is a non-negative random variable and $a>0$, then $\operatorname{Pr}(X \geq a) \leq \mathbb{E}[X] / a$.

Using Definition 1, we deduce that, given a non-negative queue length, (2b) is ensured if

$$
\bar{Q}_{k}=\lim _{T \rightarrow \infty} \frac{1}{T} \sum_{t=1}^{T} \mathbb{E}\left[Q_{k}(t)\right] \leq L_{k} \epsilon_{k}, \forall k \in \mathcal{K},
$$

is satisfied. Note that if (3) is satisfied, a certain delay performance is guaranteed (by Little's law) since the timeaveraged expected queue length will be upper bounded. Hence, in (2), we consider (3) instead of (2b). 


\section{Lyapunov Optimization Framework}

To find $\boldsymbol{\eta}(t)$ and $\boldsymbol{P}(t)$, we leverage tools from Lyapunov optimization. To satisfy (3), we introduce a virtual queue which evolves as follows:

$$
F_{k}(t+1)=\max \left\{F_{k}(t)+Q_{k}(t+1)-L_{k} \epsilon_{k}, 0\right\}, \forall k \in \mathcal{K} .
$$

Then, the conditional Lyapunov drift-plus-penalty for slot $t$ is given by:

$$
\mathbb{E}\left[\sum_{k \in \mathcal{K}}\left(\frac{F_{k}(t+1)^{2}}{2}-\frac{F_{k}(t)^{2}}{2}+\sum_{n \in \mathcal{N}} V P_{k}^{n}(t)\right) \mid \Psi(t)\right],
$$

where $\boldsymbol{\Psi}(t)=\left(Q_{k}(t), F_{k}(t), k \in \mathcal{K}\right)$ for simplicity. In (5), the non-negative parameter $V$ captures the tradeoff between power cost optimality and latency reduction. Substituting (10 and (4) into (5), and using $(\max \{x, 0\})^{2} \leq x^{2}$, we obtain

$$
\text { (5) } \begin{aligned}
\leq & +\mathbb{E}\left[\sum_{k \in \mathcal{K}} \sum_{n \in \mathcal{N}} V P_{k}^{n}(t)\right. \\
& \left.-\sum_{k \in \mathcal{K}} \tau\left(F_{k}(t)+Q_{k}(t)+\lambda_{k}(t)\right) R_{k}(t) \mid \Psi(t)\right],
\end{aligned}
$$

where $C$ is a constant. Since this constant does not affect system performance, we omit its explicit expression. The solution to (2) can be found by minimizing the upper bound in (6) in each slot, i.e.,

$$
\begin{array}{rr}
\underset{\boldsymbol{\eta}(t), \boldsymbol{P}(t)}{\operatorname{minimize}} & \sum_{k \in \mathcal{K}}\left[\sum_{n \in \mathcal{N}} V P_{k}^{n}(t)\right. \\
& \left.-\tau\left(F_{k}(t)+Q_{k}(t)+\lambda_{k}(t)\right) \mathbb{E}\left[R_{k}(t)\right]\right]
\end{array}
$$

subject to $2 \mathrm{C}-2 \mathrm{e}$,

which is an NP-hard mixed-integer programming problem. The expectation is with respect to the interference channel. Additionally, the RSU needs full global channel state information (CSI) and QSI to solve (7) in each time slot. This is clearly impractical for vehicular communication since frequently exchanging fast-varying local information between the RSU and VUE pairs incurs high overhead. To deal with this, we propose a semi-centralized approach where the RSU is leveraged.

\section{RSU-Assisted Resource Allocation}

The centralized optimization problem in (7) is challenging to solve due to the overhead caused by frequent information exchange between VUEs and the RSU at each time slot for optimal RB usage and power allocation. In this section, we propose an efficient semi-centralized approach in which we decouple the problem (7) such that the RSU performs RB allocation over a coarse timescale, and the VUE utilizes its local CSI and QSI to optimize the transmit power, at each time slot. For efficient resource allocation, the RSU needs to coordinate with the rest of the network at each time slot thus incurring a potentially large information exchange. To this end, $T_{0} \gg 1$ successive slots are assembled into one time frame to represent the coarse timescale. Hence, vehicles send their local information to the RSU at the beginning of each time frame instead of each time slot $t$. Since severe interference is caused by neighboring vehicles, in order to mitigate the interference among nearby VUEs and meet the QoS requirements as per
(3), the RSU will group vehicle pairs into a set of virtual zones 1 In view of this, we let $\mathcal{Z}$ be the set of $Z$ zones. Each zone $z \in \mathcal{Z}$ is of dynamic size and changes over time frames according to geographical proximity information of VUEs. Here, we let $\mathcal{K}_{z}$ denote the set of VUEs belonging to zone $z$. Formally, the zone formation rules are defined as:

$$
\left\{\begin{array}{l}
\left|\mathcal{K}_{z}\right| \geq 1, \forall z \in \mathcal{Z} \\
\mathcal{K}_{z} \cap \mathcal{K}_{z^{\prime}}=\emptyset, \forall z, z^{\prime} \in \mathcal{Z}, z \neq z^{\prime} \\
\bigcup_{z \in \mathcal{Z}} \mathcal{K}_{z}=\mathcal{K} .
\end{array}\right.
$$

Each VUE pair belongs to only one zone whereas each zone has at least one VUE pair. Once the zones are formed, the RSU allocates RBs to zones such that $\eta_{z}^{n} \in\{0,1\}, \eta_{z}^{n}=\eta_{k}^{n}, \forall k \in$ $\mathcal{K}_{z}$, denotes the allocation of RB $n$ to zone $z$. Let $\mathcal{N}_{z}$ be the set of orthogonal RBs assigned to each zone $z$. In each zone $z$, each VUE $k \in \mathcal{K}_{z}$ efficiently reuses the allocated RBs and optimizes its power over the given set $\mathcal{N}_{z}$ while satisfying (3). Note that $\mathcal{K}_{z}, \mathcal{N}_{z}, \eta_{z}^{n}$, and $\eta_{k}^{n}, \forall z \in \mathcal{Z}, n \in \mathcal{N}$, are static during one time frame but dynamic over frames. Given the aforementioned zone formation and RB allocation approaches, (7) can be rewritten as:

$$
\begin{array}{ll}
\underset{\mathcal{K}_{z}, \boldsymbol{\eta}, \boldsymbol{P}(t)}{\operatorname{minimize}} & \sum_{z \in \mathcal{Z}} \sum_{k \in \mathcal{K}_{z}}\left[\sum_{n \in \mathcal{N}_{z}} V P_{k}^{n}(t)\right. \\
& \left.-\tau\left(F_{k}(t)+Q_{k}(t)+\lambda_{k}(t)\right) \mathbb{E}\left[R_{k}(t)\right]\right] \\
\text { subject to } & \sum_{n \in \mathcal{N}_{z}} P_{k}^{n}(t) \leq P_{k}^{\max }, \forall t, k \in \mathcal{K}_{z}, z \in \mathcal{Z}, \\
& P_{k}^{n}(t) \geq 0, \forall t, k \in \mathcal{K}_{z}, n \in \mathcal{N}_{z}, z \in \mathcal{Z}, \\
& \sum_{z \in \mathcal{Z}} \eta_{z}^{n}=1, \forall n \in \mathcal{N}, \\
& \eta_{z}^{n} \in\{0,1\}, \forall n \in \mathcal{N}, z \in \mathcal{Z}, \\
& \text { and (8). }
\end{array}
$$

Next, we present the details of the proposed zone formation and RB allocation based on QoS requirements (average queue length constraint (3) and mean data arrival $\bar{\lambda}_{k}$ ).

\section{A. Proximity-aware Zone Formation and QoS-aware RB Al- location at the RSU}

Due to the spatio-temporal nature of $\mathrm{V} 2 \mathrm{~V}$ communication, enabling zone formation based on the spatio-temporal proximity of vehicles helps mitigate the nearby interference within each time frame. Therefore, the RSU performs zone formation while grouping physically distant VUE pairs into a set of zones such that nearby-interference is mitigated. The geographical locations of the vehicles do not change significantly during one time frame. Therefore, the latest geographical coordinates of each VUE at the beginning of every time frame for zone formation are sufficient. Let $\boldsymbol{v}_{k}$ and $\boldsymbol{v}_{k^{\prime}}$ be the geographical coordinates of the VUE pairs $k$ and $k^{\prime}$, respectively. The Euclidean distance between two pairs $k$ and $k^{\prime}$ is computed using $d_{k k^{\prime}}=\left\|\boldsymbol{v}_{k}-\boldsymbol{v}_{k^{\prime}}\right\|$. After acquiring all VUE geographical coordinates at the RSU, the following operations are performed to construct zones:

\footnotetext{
${ }^{1}$ The terms cluster and zone are used interchangeably. Moreover, in vehicle grouping, the VUE transmitter and receiver of one VUE pair are treated as a unity and grouped in the same zone.
} 


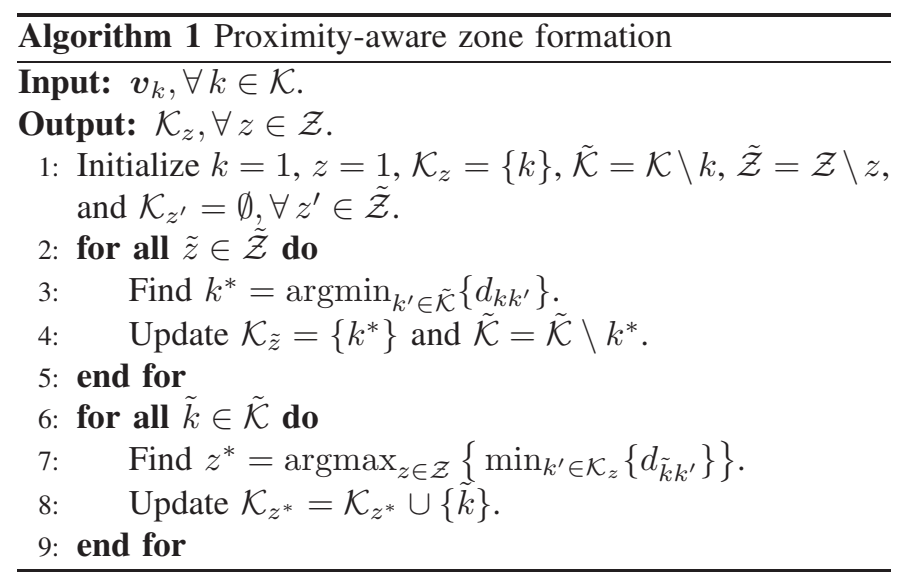

1) In order to mitigate near-by interference, RSU randomly selects one VUE pair $k$ and finds other nearest $Z-1$ pairs with respect to the Euclidean distance.

2) These $Z$ VUE pairs are separately chosen as the first composed elements of $Z$ zones.

3) Subsequently, for unsorted pairs, classify them one-byone into the farthest zone based on Euclidean distance.

Algorithm 1 describes the proposed proximity-aware zone formation method. Once zones are formed, the RSU focuses on the RB allocation. In order to satisfy the QoS requirement (3), the VUE pairs with a tighter average queue length constraint $L_{k} \epsilon_{k}$ or higher traffic demand $\bar{\lambda}_{k}$ require more RBs to release the data from their queue buffers. Therefore, considering $L_{k} \epsilon_{k}$ and $\bar{\lambda}_{k}, \forall k \in \mathcal{K}_{z}$, the RSU assigns RBs to zones in a proportional fair manner given by $\left|\mathcal{N}_{z}\right|=$ $\left(\sum_{k \in \mathcal{K}_{z}} \frac{N \bar{\lambda}_{k}}{L_{k} \epsilon_{k}}\right) /\left(\sum_{z \in \mathcal{Z}} \sum_{k \in \mathcal{K}_{z}} \frac{\bar{\lambda}_{k}}{L_{k} \epsilon_{k}}\right)$.

\section{B. Latency and Reliability-aware Power Allocation at the VUE}

Once zone formation and RB allocation (i.e., (8) and 9d) are done, each VUE $k \in \mathcal{K}_{z}$ solves (9) locally

$$
\begin{aligned}
& \underset{P_{k}^{n}(t)}{\operatorname{minimize}} \sum_{n \in \mathcal{N}_{z}} V P_{k}^{n}(t)-\sum_{n \in \mathcal{N}_{z}}\left(F_{k}(t)+Q_{k}(t)+\lambda_{k}(t)\right) \\
& \times \omega \tau \log _{2}\left(1+\frac{P_{k}^{n}(t) h_{k k}^{n}(t)}{\sigma^{2}}\right) \\
& \text { subject to } \quad \sum_{n \in \mathcal{N}_{z}} P_{k}^{n}(t) \leq P_{k}^{\max }, \\
& P_{k}^{n}(t) \geq 0, \forall n \in \mathcal{N}_{z},
\end{aligned}
$$

which must be solved at each time slot $t$. Here, the aggregate interference is negligible since orthogonal RBs are allocated to adjacent VUE pairs. Applying the Karush-KuhnTucker (KKT) conditions to the convex optimization problem (10), the VUE transmitter finds optimal the transmit power $P_{k}^{n *}(t), \forall n \in \mathcal{N}_{z}$, which satisfies

$$
\frac{\left(F_{k}(t)+Q_{k}(t)+\lambda_{k}(t)\right) \omega \tau h_{k k}^{n}(t)}{\left(\sigma^{2}+P_{k}^{n *}(t) h_{k k}^{n}(t)\right) \ln 2}=V+\gamma
$$

if $\frac{\left(F_{k}(t)+Q_{k}(t)+\lambda_{k}(t)\right) \omega \tau h_{k k}^{n}(t)}{\sigma^{2} \ln 2}>V+\gamma$. Otherwise, $P_{k}^{n *}(t)=0$. Moreover, the Lagrange multiplier $\gamma$ is 0 if $\sum_{n \in \mathcal{N}_{z}} P_{k}^{n *}(t)<P_{k}^{\max }$, and we have $\sum_{n \in \mathcal{N}_{z}} P_{k}^{n *}(t)=$ $P_{k}^{\max }$ when $\gamma>0$. After sending information, VUE pair $k$

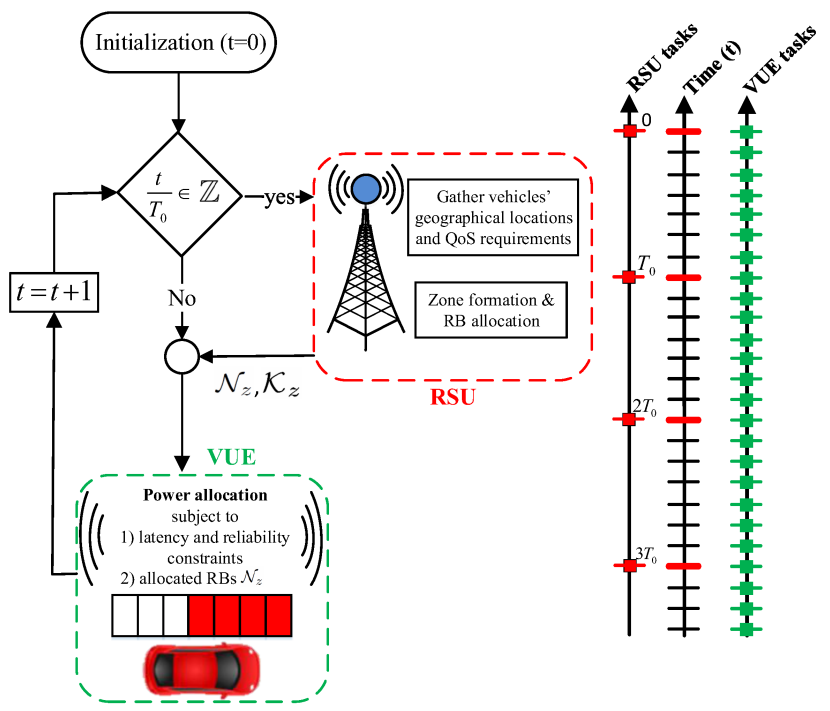

Figure 2. Flow diagram of the proposed hierarchical resource allocation scheme.

updates $Q_{k}(t+1)$ and $F_{k}(t+1)$ as per (1) and (4). The detailed steps of the proposed framework are summarized in Fig. 2

\section{NumericAl Results}

In this section, we simulate a Manhattan mobility model with different vehicle densities that are uniformly distributed over a $250 \times 250 \mathrm{~m}^{2}$ area. As shown in Fig. 1 four buildings with $100 \mathrm{~m}$ breadth are deployed and spaced by two lanes for bi-directional traffic mobility. Vehicles travel along defined roads with an average speed of $50 \mathrm{~km} / \mathrm{h}$. The safety distance between the VUE transmitter and VUE receiver dynamically ranges from $10 \mathrm{~m}$ to $20 \mathrm{~m}$. For V2V communication, we consider the Berg recursive path loss model [10] and lineof-sight propagation within each VUE pair. For all VUE pairs $k \in \mathcal{K}$, we set $P_{k}^{\max }=10 \mathrm{dBm}, \bar{\lambda}_{k}=200 \mathrm{kbps}$, $L_{k}=2000 \mathrm{bits}$, and $\epsilon_{k}=0.1$. The other parameters are: $N=15 \mathrm{RBs}, \omega=180 \mathrm{kHz}, \tau=1 \mathrm{~ms}, \sigma^{2}=-80 \mathrm{dBm}$, $T_{0}=100$, and $Z=5$ zones. For the baseline, we refer to the configuration 1 in 3GPP [11]. Therein, each VUE optimizes its transmit power over all RBs in each time slot. We use $Q(t) / \lambda$ as the instantaneous queuing latency metric [12].

The tradeoff between the average queuing latency and the average power consumption is studied in Fig. 3 for different densities of VUE pairs. When $V$ is small as per (10a), the VUE focuses on the rate maximization to decrease its queuing latency which consumes more power. Oppositely, for a large $V$, the VUE saves power consumption by allowing the queue length to grow. Fig. 3 shows that the proposed approach achieves not only a queuing latency requirements of $0.125 \mathrm{~ms}$ [4] but also an average queuing latency reduction of up to $97 \%$ as compared to the baseline for different vehicle densities. Furthermore, the average latency enhancement is more prominent when $K>N$ due to the RSU-aided interference mitigation.

Subsequently, considering the case $V=0$ which has the lowest queuing latency, we investigate the transmission reliability via the complementary cumulative distribution function 


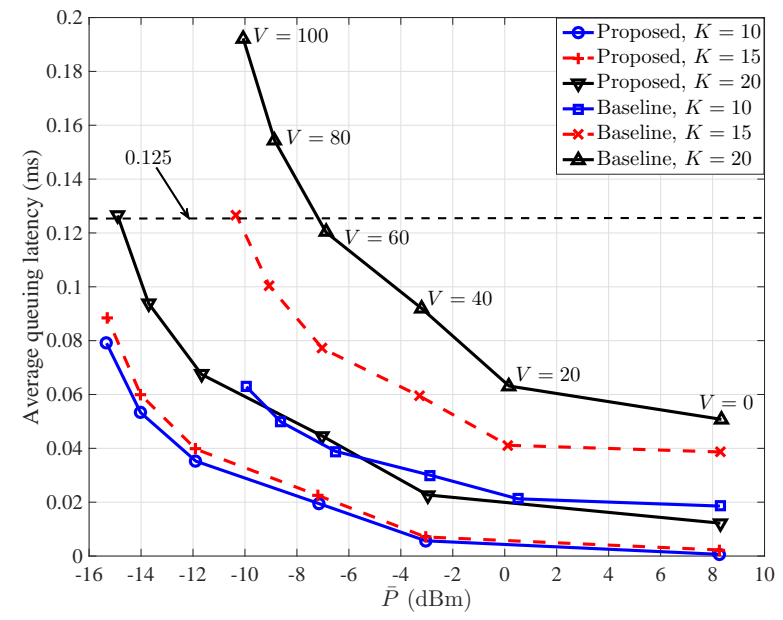

Figure 3. Tradeoff between average queuing latency and average power for various densities of VUEs.

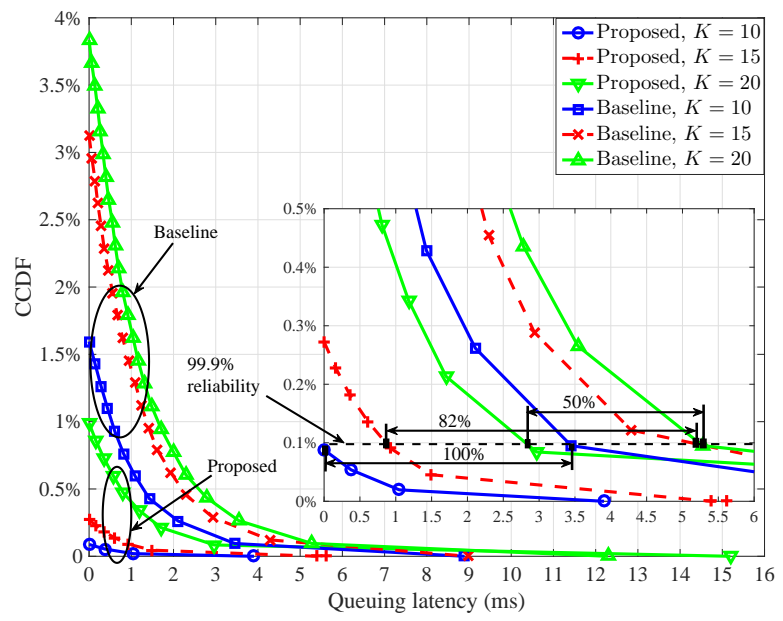

Figure 4. CCDF of the instantaneous queuing latency for various densities of VUEs with $V=0$.

(CCDF) of the instantaneous queuing latency. The reliability performance for varying VUE densities and different reliability constraint values $\epsilon$ is shown in Fig. 4 and Fig. 5, respectively. It can be seen that, in different network settings, our approach always satisfies the aforementioned reliability constraint as per (2b) and achieves higher reliability performance (i.e., lower CCDF values) compared with the baseline. Further, for the 99.9\% reliability case, i.e., $0.1 \%$ of the CCDF value, in Fig. 4 and Fig. 5, our proposed approach achieves a queuing latency reduction up to $100 \%, 82 \%$, and $50 \%$ for $K=10,15$, and 20 , respectively, as compared to the baseline. Finally, Fig. 5 shows that, in order to achieve $99.9 \%$ reliability for different values of $\epsilon$, the proposed approach reaches up to $73 \%$ and $50 \%$ for $\epsilon=0.01$ and 0.1 , respectively, as compared to the baseline.

\section{CONCLUSiOnS}

In this paper, we have studied the performance of $\mathrm{V} 2 \mathrm{~V}$ communication in terms of queuing latency and reliability. The network objective is to minimize VUEs' power consumption subject to queuing latency and reliability constraints modeled as probability constraints on data queue length. We have proposed a novel two-timescale resource allocation approach. In the slow timescale, the RSU forms virtual zones and

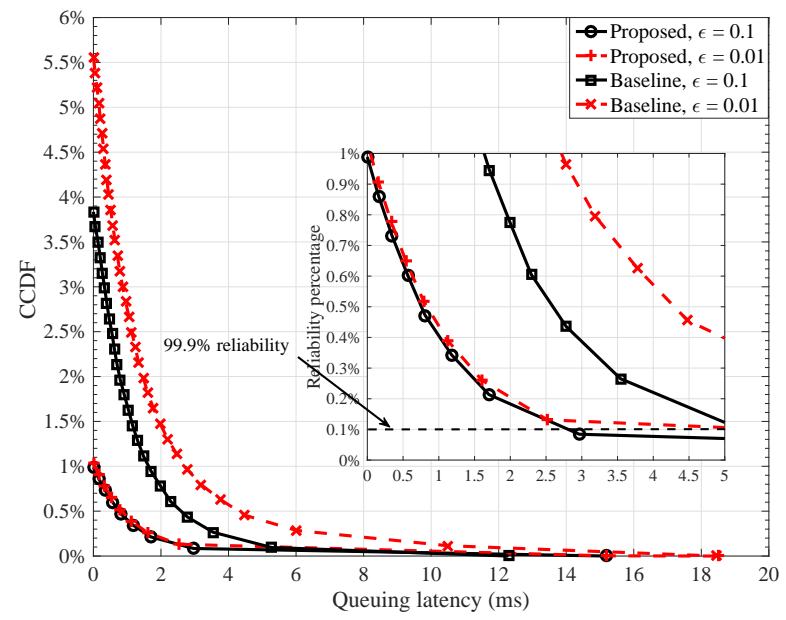

Figure 5. CCDF of the instantaneous queuing latency for different $\epsilon$ with $K=20$ VUE pairs and $V=0$.

allocates RBs to zones based on vehicles' spatial information and QoS requirements. Using Lyapunov optimization, every vehicle locally optimizes its transmit power while satisfying queuing latency and reliability constraints. Simulation results have shown that our proposed scheme outperforms the baseline with an average queuing latency reduction up to $97 \%$ and achieves significant improvement in terms of reliability.

\section{ACKNOWLEDGMENTS}

This research was supported by the Academy of Finland project CARMA, the NOKIA donation project FOGGY, the Thule Institute strategic project SAFARI, and the U.S. Office of Naval Research (ONR) under Grant N00014-15-1-2709.

\section{REFERENCES}

[1] G. Araniti, C. Campolo, M. Condoluci, A. Iera, and A. Molinaro, "LTE for vehicular networking: A survey," IEEE Commun. Mag., vol. 51, no. 5, pp. 148-157, May 2013.

[2] ETSI EN Std 302 637-2, "Intelligent transport systems; Vehicular communications; Basic set of applications; Part 2: Specification of cooperative awareness basic service," v. 1.3.0, Aug. 2013.

[3] A. Vinel, “3GPP LTE versus IEEE 802.11p/WAVE: Which technology is able to support cooperative vehicular safety applications?" IEEE Wireless Commun. Lett., vol. 1, no. 2, pp. 125-128, Apr. 2012.

[4] Nokia, "5G highlights," 5G Technology Workshop - Potential Technology for 3GPP Rel-15, Oct. 2016.

[5] A. Anpalagan, M. Bennis, and R. Vannithamby, Design and Deployment of Small Cell Networks. Cambridge University Press, Dec. 2015.

[6] W. Sun, E. G. Ström, F. Brännström, K. C. Sou, and Y. Sui, "Radio resource management for D2D-based V2V communication," IEEE Trans. Veh. Technol., vol. 65, no. 8, pp. 6636-6650, Aug. 2016.

[7] M. Botsov, S. Stańczak, and P. Fertl, "Optimized zone design for location-based resource allocation in mobile D2D underlay networks," in Proc. Int. Symp. Wireless Commun. Syst., Sep. 2016, pp. 175-181.

[8] S. Zhang, Y. Hou, X. Xu, and X. Tao, "Resource allocation in D2Dbased V2V communication for maximizing the number of concurrent transmissions," in Proc. IEEE 27th Annu. Int. Symp. Pers., Indoor, Mobile Radio Commun., Sep. 2016, pp. 1-6.

[9] J. D. C. Little, "A proof for the queuing formula: $L=\lambda W$," Operations Research, vol. 9, no. 3, pp. 383-387, 1961.

[10] Scenarios, requirements and KPIs for $5 \mathrm{G}$ mobile and wireless system, ICT-317669-METIS/D1.1, METIS deliverable D1.1, Apr. 2013.

[11] 3rd Generation Partnership Project, "3GPP initial cellular V2X standard, work item RP-161894,” Tech. Rep., Sep. 2016.

[12] M. Molina, O. Muñoz, A. Pascual-Iserte, and J. Vidal, "Joint scheduling of communication and computation resources in multiuser wireless application offloading," in Proc. IEEE 25th Annu. Int. Symp. Pers., Indoor, Mobile Radio Commun., Sep. 2014, pp. 1093-1098. 